\title{
Rheological Modelling of Bio-fluids Using Moving Coil Transducers
}

\author{
Ali Umit Keskin, Feride Sermin Utku \\ Department of Biomedical Engineering, Yeditepe University, 34755, Istanbul, Turkey. \\ auk@yeditepe.edu.tr; bilgenfe17@yahoo.com
}

\section{Extended Abstract}

Blood rheology is the study of flow properties of plasma and cells that may alter in disease processes. Rheometers can be of very simple or complex structures in construction, depending on the type of application they are used [1]. Hemorheology allows only certain types of equipment to be used in biomedical applications.

Some researchers [2] used impedance spectroscopy as a means of analysis for the rheological properties of a liquid, using a mixture of Genetic and the Nelder-Mead simplex algorithm to obtain the equivalent circuit parameters of the measurement system (vibrating wire method). In our project (presented here), we use a rather straightforward procedure, based on the electrical equivalent circuit synthesis and realization for moving coil transducers that can be performed using a model based circuit topology, since this equivalent circuit can be analysed as a driving point impedance where . Thiele parameters" constitute the equivalent circuit elements.

In this work, we present the results of our laboratory measurements using a moving coil transducer and various fluids. Equivalent circuit parameters were obtained by measuring and recording the impedance versus frequency data. All measurements were conducted around the same temperature $\left(24^{\circ} \mathrm{C}\right)$. Calibration measurements are performed in air using an empty polystyrene cup in a test box, and using a moving coil transducer with hollow spindle attachment. Then, similar measurements and recordings are repeated for a test cup filled with distilled water as the second calibration fluid, and various test fluids mimicking blood and plasma [3]. Driving point impedance functions for each case are calculated based on the results of these measurements. Finally, Thiele model parameters are implemented to set up driving point impedance functions for each corresponding measurement case. It is to be noted here that, there is only a slight difference between measured and computed vibration magnitude spectral results. The small difference is the observed weak amplitude peak at $\mathrm{f}=3 \mathrm{kHz}$ in measurements, which does not appear in computationally obtained spectra.

We noted that temperature is an important factor in this kind of measurement. We used a method to interpolate reported water viscosity values. Provision of Standard Viscosity samples greatly improves quantitative studies. Similar data is required for any fluid sample. This can be advantageous to re-scale the equipment for viscosity measurements performed at different temperatures. Therefore, an empirical equation will be more reliable with the addition of temperature dependence parameter.

It has been noted that there are meaningful relationships between measured parameters and the polynomial coefficients of synthesized third order driving point impedance functions. Preliminary results of these experiments have been found to be in good agreement with theory.

\section{References}

[1] A. Y. Malkin, A. I. Isayev, Rheology, Concepts, Methods, and Applications. 2nd Edition. ChemTec Publishing, 2012.

[2] F. M. Janeiro, P. M. Ramos, J. M. N. A. Fareleira, J. C. F. Diogo, D. R. C. Máximo, and F. J. P. Caetano, "Impedance Spectroscopy of a Vibrating Wire for Viscosity Measurements," Instrumentation and Measurement Technology Conference (I2MTC 2010) IEEE, pp. 1067 - 1072, 2010.

[3] K. Brookshier and J. Tarbell, "Evaluation of a transparent blood analog fluid: Aqueous Xanthan gum/glycerin," Biorheology, vol. 2, pp.107-116, 1993. 